\title{
Investigation of genotoxic and cytotoxic effects of acrylamide in HEK293 cell line
}

\begin{abstract}
Background/aim: Acrylamide is a cytotoxic, genotoxic and neurotoxic chemical for human. High level of acrylamide uptake causes genotoxic and neurotoxic effects, however the cellular damage mechanisms of long-term low-dose acrylamide uptake are not fully known yet. The present study investigated the cytotoxic and genotoxic effects of acrylamide on the HEK293 cells
\end{abstract}

Materials and methods: Genotoxic effects of acrylamide were examined by micronuclei formation assay and its impact on the cell viability was measured by MTT reduction assay. For studying genotoxicity and determining the source of the micronucleus, FISH (Fluorescent in Situ Hybridization) assay was applied. The effect of acrylamide on oxidative stress, as well as oxidative stress pathway markers such as glutathione (GSH), superoxide dismutase (SOD) and catalase (CAT) were investigated.

Results: Acrylamide reduced cell viability significantly. Radical oxygen species formed by the metabolism of acrylamide has increased oxidative stress in cells and the amount of SOD significantly decreased. The amount of GSH decreased in proportion to the increase in the amount of hydrogen peroxide and the level of oxidized GSH (GSSG) has declined.

Conclusion: Our work has supported that the induction of oxidative stress causes cell death and carcinogenesis. Consequently acrylamide, for the HEK293 cell line is shown to be a cytotoxicity, genotoxicity, and oxidative stress enhancer.

Keywords: acrylamide, cytotoxicity, genotoxicity
Volume 9 Issue 5 - 2018

\author{
Fatma Secer Celik,' Tulin Cora, ${ }^{2}$ Aysel Kalayci \\ Yigin ${ }^{3}$ \\ 'Meram Medical Faculty, Department of Medical Biology, \\ Necmettin Erbakan University, Turkey \\ Medical Faculty, Department of Medical Genetics, Selcuk \\ University, Turkey \\ ${ }^{3}$ Deparment of Medical Genetics, Istanbul University, Cerrahpasa \\ Medical School,Turkey
}

Correspondence: Fatma Secer Celik, Meram Medical Faculty, Department of Medical Biology, Necmettin Erbakan University, 42090 Meram, Konya, Turkey, Tel 0090-5305927728, Email bio.sacer@gmail.com

Received: October 24, 2018 | Published: October 31, 2018

\section{Introduction}

Acrylamide or acrylic amide is a chemical compound of $\mathrm{C}_{3} \mathrm{H}_{5} \mathrm{NO}$. Acrylamide, a vinyl polymer and well soluble in water, is a chemical used to make polyacrylamide materials. Acrylamide was originally synthesized by chemically and started to be used in a wide variety of industrial fields, such as the textile, paper, and cosmetic industries.

Until 2000, the knowledge that acrylamide was not formed naturally but could be chemically synthesized was accepted. Tareke and colleagues from Stockholm University have announced to the scientific world that abundant amounts of acrylamide are formed when food is cooked at temperatures above $120^{\circ} \mathrm{C}$. ${ }^{1,2}$ The amount of acrylamide present during the cooking process is increasing in direct proportion to the temperature and duration of exposure. The first study of how much acrylamide we take on a daily basis after being detected in acrylamide-cooked foods was done in the Netherlands. According to this study, human subjects fed with foods cooked at high temperatures were found to take $0.5 \mathrm{mg} / \mathrm{kg}$ of acrylamide on average per day depending on their body weight. ${ }^{3}$ However, in recent studies it has been reported that this amount is $0.27 \mu \mathrm{g} /$ $\mathrm{kg}$ for female individuals and $0.36 \mu \mathrm{g} / \mathrm{kg}$ for male individuals in the Dutch population. ${ }^{4}$ Similar to these results, in the American population study this amount was found to be $0.44 \mu \mathrm{g} / \mathrm{kg}$ per person per day. ${ }^{5}$

Food-borne acrylamide occurs as a result of the Maillard reaction between monosaccharides such as fructose or glucose and amino acids such as asparagine at temperatures above $120^{\circ} \mathrm{C} .{ }^{6}$ In particular, asparagine plays a key role in the formation of acrylamide from the standard 20 amino acids that go into the structure of proteins. No acrylamide formation during the scalding of food.

Experimental studies in which acrylamide was given to mice and rats showed that glycidamide was abundant in acrylamide and glycinamide interferes with the purine bases of liver, lung and kidney DNA of these animals, leading to genotoxic effects. ${ }^{8,9}$ It has been determined that the glycidamide administered to animals is more mutagenic than the same dose of acrylamide. ${ }^{10,11}$ Cells treated with glycidamide or acrylamide showed more Adenine-Guanine transitions and Guanine-Cytosine transpositions than control cells that were spontaneously mutated. Guanine-Timin transverses in the cells treated with glycidamide occurred at much higher levels. Studies have shown that the mutagenesis of acrylamide in human and mouse cells results from the promutagenic binding capacity of acrylamide metabolites and glycidamide to DNA..$^{9,12}$

Acrylamide exhibits cytotoxic and genotoxic effects in some tissue cells. It was also found that the in vivo studies were neurotoxic and tumorigenic. However, dietary acrylamide is far below the doses used in these studies and is long-lasting. Based on this, this study was conducted in order to investigate the mechanism by which low levels of acrylamide are used to cause cancer. In this study, we have investigated the levels 
of oxidative stress modulators in our study when we tried to avoid acrylamide by causing oxidative stress to disturb cellular equilibrium and causing cancerogenesis. ${ }^{13}$

\section{Materials and methods}

\section{a. Chemicals}

All cell culture reagents and buffers were obtained from Gibco (Rockville, MD, USA), GSH/GSSG Assay Kit, Superoxide Dismutase Assay Kit and Catalase Assay Kit from Bioassays (Hayward, USA) and Acrylamide from Sigma Aldrich (St Louis, MO, USA) Company.

\section{b. Cell culture}

HEK293 human embryonic kidney cell line was obtained from ATCC (American Type Culture Collection) and cultured in RPMI 1640 medium supplemented with $10 \%$ fetal bovine serum (Biochrome, Germany), 1\% L-glutamine and 1\% streptomycin-penicillin (Biochrome, Germany), at $37^{\circ} \mathrm{C}$ in a $5 \% \mathrm{CO}_{2}$ humidified atmosphere.

\section{c. Assessment of cell viability}

Cell viability analysis carried out by MTT (Thiazolyl Blue Tetrazolium Bromide) assay. For this purpose, approximately 104 cells added to $100 \mu \mathrm{l}$ culture medium and incubated in 96 well-plate at $37^{\circ} \mathrm{C}$ in $5 \% \mathrm{CO}_{2}$. The cells treated with $100 \mu \mathrm{l}$ of the medium that contains various concentrations of acrylamide for 24, 48 and $72 \mathrm{~h}$. Then, $10 \mu 112 \mathrm{mM}$ Thiazolyl Blue Tetrazolium Bromide (MTT; Sigma Aldrich®M2128) solution added to each well and incubated for $4 \mathrm{~h}$. Absorbance was measured at $490 \mathrm{~nm}$ wavelength in the microplate reader (Biotek, USA).

\section{d. Genotoxic activity analysis}

The genotoxic effect of the acrylamide HEK293 cell line was assessed by Cytokine-Blocking Micronucleus Assay. $2.5 \times 10^{3}$ cells were plated in T25 culture flasks and incubated for 48 hours. Acrylamide medium was added to the cells at different concentrations $(0.1,0.01,0.001 \mathrm{M})$ and incubated for 24 hours. On the $48^{\text {th }}$ hour, cytochalasin-B was added with fresh medium and incubated for 24 hours. Cells were removed by trypsinization, washed, fixed on dry slides with cold methanol after the necessary centrifugation steps. Giemsa staining was then carried out after the dry period and then counting by light microscopy. For MN frequency; 1000 binucleated cells were counted and the ratio of MN/1000BN (number of micronucleus/ number of cells of 1000 Binucleotides) was taken.

\section{e. Fluorescence in Situ Hybridization (FISH) technique}

Preparations prepared were subjected to FISH (Fluorescent in Situ Hybridization) using probes (Cytocell) of all centromere gene regions.

The preparations were examined using appropriate filters on a Nikon ECLIPSE E 600 fluorescence microscope. Nuclei containing FISH signals were analyzed and photographed.

\section{f. Investigation of cell redox modulator levels}

The EnzyChrom ${ }^{\text {TM }}$ GSH/GSSG Assay Kit for GSH, the EnzyChrom $^{\text {TM }}$ Superoxide Dismutase Assay Kit for SOD, and the EnzyChrom ${ }^{\mathrm{TM}}$ Catalase Assay Kit for CAT were used for quantitation of redox modulators in the acrylamide-treated cells. Procedures for each site were followed. The data was analyzed.

\section{g. Statistical analysis}

Statistical analyses were performed using GraphPad Prism 6.0 software (GraphPad Software Inc., La Jolla, CA, USA). Intragroup comparisons investigated by using dependent t-test and difference between groups investigated by ANOVA test. Tests considered a basic significance level of $\mathrm{p}<0.05$.

\section{Results}

\section{a. Acrylamide reduces viability and cell proliferation}

MTT method was used for viability analysis of the cells. Acrylamide doses of 2, 1, 0.1, 0.01, 0.001 $\mathrm{M}$ were selected in accordance with the literature to be used in the study, and the determined doses were administered to the cells for 24, 48 and 72 hours. The results are shown in Figure 1. According to the results obtained, the ICF was determined to be $0.01 \mathrm{M}$.

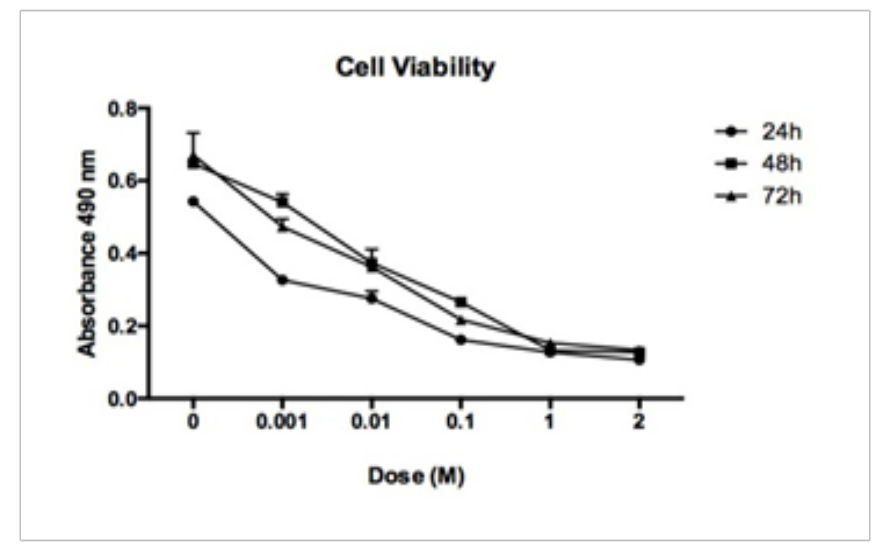

Figure I 24 hrs, 48 hrs, 72 hrs MTT result plots of HEK293 cells treated with acrylamide.

The activity of acrylamide in HEK293 cells was found to decrease at $24(p=0.0004), 48(p=0.0060)$ and $72(p=0.0220)$ hours, respectively, when compared to the control without acrylamide (Figure 1). This significant reduction clearly demonstrated the negative and proliferative effect of acrylamide on cell viability. To study this situation genotoxically, we continued to work with the cytokine-blocked micronucleus test.

\section{b. Acrylamide is genotoxic}

The MTT test was used to determine the ICF and the micronucleus test was applied to this dose with the appropriate new dose selections. 2 and $1 \mathrm{M}$ doses, which greatly reduce cell viability in the first dose selection, were not used in micronuclei because the cell volume was difficult to quantify. The resulting micronucleus samples are shown in Figure 2.

The total number of cells counted according to doses, the 
only cytoplasmic and double nucleated cells that formed, and those containing micronuclei were counted (Table 1). The number of binucleuses was fixed at 1200 and the proportion was made accordingly.

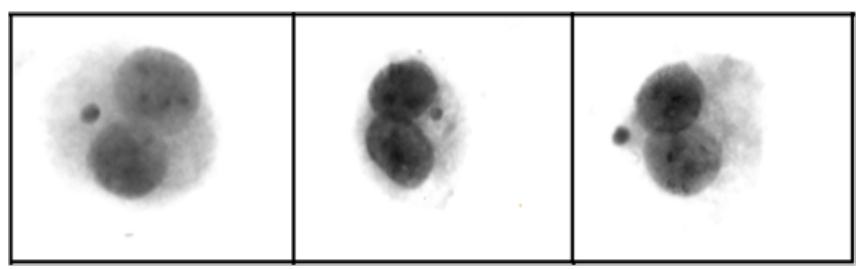

Figure 2 The micronucleus formed in the HEK293 cell line treated with acrylamide.

Table I The number of micronuclei formed in the Hek293 cell line at certain doses of acrylamide

\begin{tabular}{llll}
\hline Dose & Total Cell Count & Bınucleı Cells & Micronucle Cells \\
\hline Control & 2500 & 1200 & 2 \\
0,001 & 2780 & 1200 & $1 \mathrm{I}$ \\
0,01 & 1830 & 1200 & 38 \\
0,1 & 1200 & 1200 & 47 \\
\hline
\end{tabular}

After micronucleus counts of acrylamide-treated cells at different doses, micronucleus frequencies were calculated (Table 2) and statistical calculation was performed.

Table 2 Micronuclei Frequencies

\begin{tabular}{ll} 
Dose & MN Frequencies \\
\hline Control & $20 / 1200=0,0016$ \\
0,001 & $11 / 1200=0,0091$ \\
0,01 & $38 / 1200=0,031$ \\
0,1 & $47 / 1200=0,039$ \\
\hline
\end{tabular}

The number and frequency of micronuclei formed due to the increase in dose are increased.

\section{c. Acrylamide cuses DNA fractures}

Results obtained by micronucleus test were confirmed by FISH technique. According to this technique, it was taken into account that micronuclei were not marked with the centromere probe. Marked micronuclei were assessed as having a centromere region. Thus, a chromosomal source of micronuclei was detected (Figure 3).

\section{d. Acrylamide changes levels of cell redox modulators}

SOD allows endogenous or exogenous reaction of reactive oxygen species in the cell to $\mathrm{H}_{2} \mathrm{O}_{2}$ and participates in other reactions. When doing this, the SOD capacity decreases. According to our findings, acrylamide decreased the amount of SOD in the cell depending on the dose (Figure 4).

The $\mathrm{H}_{2} \mathrm{O}_{2}$ formed by SOD in the cell is rendered inactive by being converted to water by CAT and GPX. GSH reacts with $\mathrm{H}_{2} \mathrm{O}_{2}$ to form GSSG which is the oxidized glutathione. The amount of GSH, which decreases in amount, causes the amount of GSSG to increase. According to our findings, we obtained the expected result, and the amount of GSSG increased as the amount of GSH decreased as much as the dose of IC50 (Figure 5). However, from the IC50 dose, the amount of the oxidized glutathione has also been effectively reduced. This is discussed in the discussion of the change in the amount of CAT.
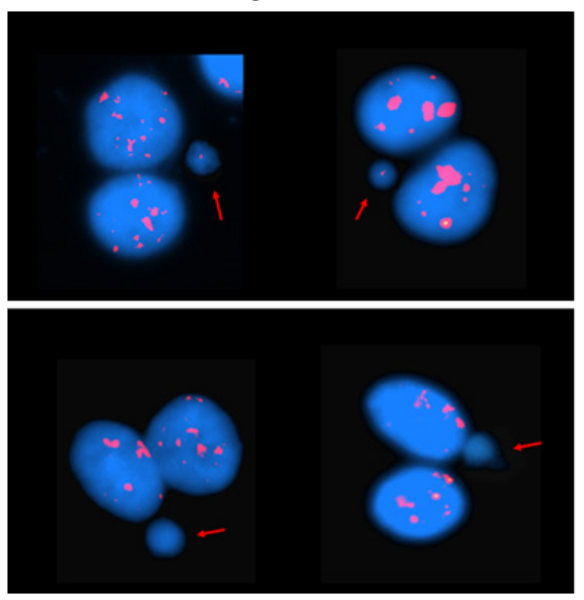

Figure 3 (A) Centric Micronuclei (B) Acentric Micronuclei.

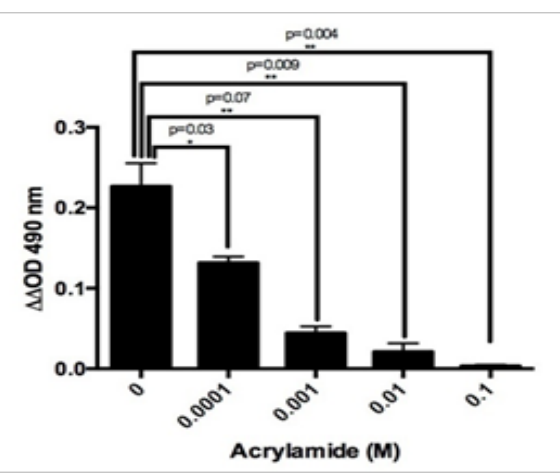

Figure 4 Changes in the amount of SOD depending on the doses of acrylamide.
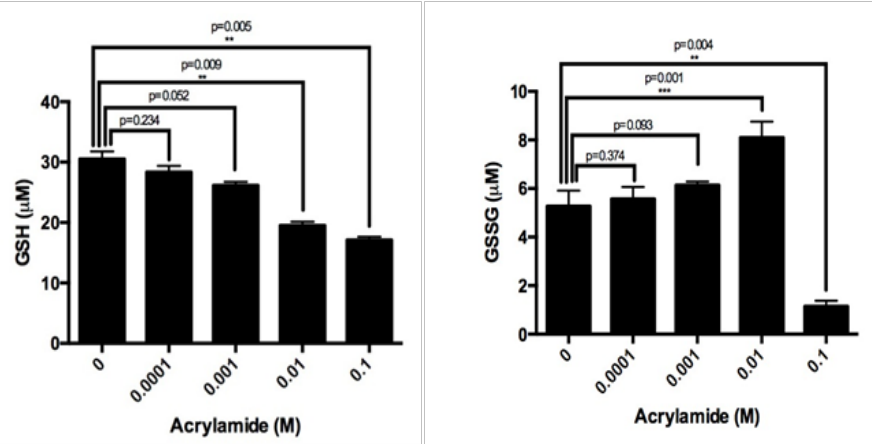

Figure 5 Change in the amounts of GSH and GSSG according to the applied acrylamide doses.

$\mathrm{H}_{2} \mathrm{O}_{2}$, which is increased in the cell by SOD, is firstly trapped by GSH and CAT, while GSH is converted into the oxidant form, CAT turns $\mathrm{H}_{2} \mathrm{O}_{2}$ into the water by one reaction. When different doses of acrylamide were administered, the amount of CAT increased due to the dose but decreased from the IC50 dose (Figue 6). This decline is due to the same steps as GSH. 


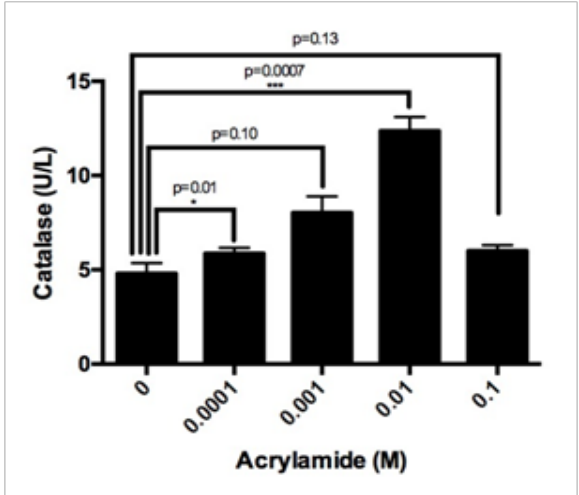

Figure $\mathbf{6}$ Change in amount of CAT depending on the dose of acrylamide applied.

\section{Discussion}

Acrylamide is a xenobiotic that is commonly absorbed by humans through skin absorption and oral. Since acrylamide was not known to be food-borne until 2002, only occupational exposure studies were conducted. Later, its use in cosmetic products has brought about the uptake of xenobiotics into the body. Xenobiotics are also important for their formation of harmful metabolites after they have been metabolized. When acrylamide is taken into the cell, it transforms itself into a more active metabolite, glycidamide. Studies have shown that glycidamide increases genotoxicity. ${ }^{14-16}$

In this study, firstly, we examined how acrylamide effects viability in the HEK293 cell line. According to the MTT test results, when an amount of acrylamide increased the cell viability significantly decreased. The decrease of cell viability can be attributed to many reasons. These include damage to the genetic material of the cell, impaired oxidative stress balance in the cell. In the study of Manjanatha, acrylamide, and glycidamide, which participate in the drinking water of rats, have been shown to increase cell mutations 2-5 times in the lung. According to this study, A: T -> T: A, G: C -> C: G transversions and $+1 /-1$ frame shift mutations were detected. ${ }^{17}$ Recent studies have shown that the genotoxic (mutagenic) action of acrylamide and glycidamide on cell DNA results in very serious damage such as breaks in the DNA chain, cross-chain linkages, base shifts and base changes. These genotoxic effects on the DNA have been reported to be caused by glycidamide, an acrylamide metabolite largely present in acrylics. Glycidamide and DNA incorporation studies showed GA-DNA incorporation even at very low doses such as $1 \mu \mathrm{M}$ as determined by HPLC/MS method. ${ }^{16}$

To examine the genotoxic effect of acrylamide on cells, we performed a cytokine-blocked micronucleus test. The cytokineblocked micronucleus method is primarily used to measure cytogenetic damage. In this study, acrylamide increased the cytotoxic effect and the cell viability decreased. In acrylamidetreated cells which under ICF was showed micronucleus formation. Cell amount decreased as low as not allowed to have cell count at doses with high cytotoxic effect.
FISH method was used to identify both the micronucleus formation and the source of micronuclei. According to the FISH results, the number of micronuclei carrying centromere was three times lower than non-carryings. This suggests that acrylamide causes breaks in DNA, causing DNA breaks and thus genotoxic effect. In addition, acrylamide acts on spindle cells during cell division, separating the chromosomes or chromosomal fragments, suggesting a micronucleus structure with the centromere region. This suggests that acrylamide increases the formation of aneuploidy in the cells. Högstedt and Karlsson developed the aneuploid-inducing agents with the modified methods and differentiated the clastogens with $\mathrm{MN}$ size difference; showed that MNs that were induced by clastogens were larger in size, containing small, and genesinduced MNs containing full chromosomes, while those containing acentric chromosomal fragments. ${ }^{18}$

Oxidative stress is a condition in which the oxidant/ antioxidant balance is degraded in favor of oxidants as a result of an excessive increase of SORs in cells or a decrease in the level of antioxidants. In oxidative stress; Atherosclerosis, fever, diabetes, ischemia/reperfusion injury, gene mutation, cancer development, and xenobiotic damage to tissues. ${ }^{19,20}$ In our studies have shown that acrylamide has an effect on cell viability and it has been discussed how low doses can be effective in cellular mechanisms. Oxidative stress pathway was thought to be a critical pathway for xenobiotics and oxidative stress markers such as GSH, SOD and CAT activities were also investigated. In our study, after the treated of acrylamide to HEK293 cells, the amount of SOD decreased. Reactive oxygen generated by metabolizing acrylamide into the cell is captured by SOD and converted to hydrogen peroxides. The decrease in the amount of total GSH in relation to the dose of acrylamide increased in the amount of oxime GSH (GSSG) up to the IC50 dose, then decreased. In a similar way, the amount of CAT increased up to the IC50 dose and then decreased. Taken together, the oxidative stress induced by acrylamide was first inactivated by GSH, in addition to the increased amount of CAT in the cell but not to the IC50 dose. It has been found that the excessive decrease of cellular GSH capacity triggers a second pathway, CAT reactions, and that the total CAT amount decreases. According to these results, the metabolism of acrylamide shows that it is first taken up by SOD, converted to hydrogen peroxides and then metabolized through GSH and CAT pathways. Increasing the dose used leads to oxidative stress by disrupting the oxidant/antioxidant balance. This causes a decrease in cell viability, increase in cell death and tumorigenesis. Our work has supported that the induction of oxidative stress causes cell death and carcinogenesis.

\section{Acknowledgements}

This study was supported by the Selcuk University Research Fund with the project number 14202016.

\section{Conflict of interest}

The authors declare there is no conflict of interest. 


\section{References}

1. Tareke E, Rydberg P, Karlsson P, et al. Acrylamide: a cooking carcinogen. Chemical Res Toxicol. 2000;13(6):517-522.

2. Tareke E, Rydberg P, Karlsson P, et al. Analysis of acrylamide, a carcinogen formed in heated foodstuffs. J Agri Food Chem. 2002(17);50:4998-5006.

3. Boon PE, de Mul A, van der Voet H, et al. Calculations of dietary exposure to acrylamide. Mutation Research. 2005;580(1-2):143-155.

4. Jakobsen LS, Granby K, Knudsen VK, et al. Burden of disease of dietary exposure to acrylamide in Denmark. Food Chem Toxicol. 2016;90:151-159.

5. Doerge DR, Young JF, Chen JJ, et al. Using dietary exposure and physiologically based pharmacokinetic/pharmacodynamic modelling in human risk extrapolations for acrylamide toxicity. J Agric Food Chem. 2008;56(15):6031-6038.

6. Stadler RH, Blank I, Varga N, et al. Acrylamide from Maillard reaction products. Nature. 2002;419(6906):449-450.

7. Mottram DS, Wedzicha BL, Dodson AT. Food chemistry: Acrylamide is formed in the Maillard reaction. Nature. 2002;419:448-449.

8. Segerbäck D, Calleman CJ, Schroeder JL, et al. Formation of /V-7(2-carbamoyl-2-hydroxyethyl)guanine in DNA of the mouse and the rat following intraperitoneal administration of [14C]acrylamide. Carcinogenesis. 1995;16(5):1161-1165.

9. Gamboa da Costa G, Churchwell MI, Hamilton LP, et al. DNA adduct formation from acrylamide via conversion to glycidamide in adult and neonatal mice. Chem Res Toxicol. 2003;16(10):1328-1337.

10. Sumner SC, Fennell TR, Moore TA, et al. Role of cytochrome P450 2E1 in the metabolism of acrylamide and acrylonitrile in mice. Chem Res Toxicol. 1999;12(11):1110-1116.

11. Favor J, Shelby MD. Transmitted mutational events induced in mouse germ cells following acrylamide or glycidamide exposure. Mutat Res. 2005;580(1-2):21-30.

12. Besaratinia A, Pfeifer GP. Genotoxicity of acrylamide and glycidamide. J Natl Cancer Inst. 2004;96(13):1023-1029.

13. Kuperman AS. Effects of acrylamide on the central nervous system of the cat. J Pharmacol Exp Ther. 1958;123(3):180-192.

14. Fuhr U, Boettcher MI, Kinzig-Schippers M, et al. Toxicokinetics of acrylamide in humans after ingestion of a defined dose in a test meal to improve risk assessment for acrylamide carcinogenicity. Cancer Epidemiol Biomarkers Prev. 2006; 15(2):266-271.

15. Mucci LA, Wilson KM. Acrylamide intake through diet and human cancer risk. J Agric Food Chem. 2008;56(15):6013-6019.

16. Bandarra S, Fernandes AS, Magro I, et al. Mechanistic insights into the cytotoxicity and genotoxicity induced by glycidamide in human mammary cells. Mutagenesis. 2013;28(6):721-729.

17. Manjanatha MG, Li-Wu Guo, Sharon D, et al. Acrylamide-Induced Carcinogenicity in mouse Lung Involves Mutagenicity: cII gene mutations in the Lung of Big BlueMice Exposed to Acrylamide and Glycidamide for up to 4 Weeks. Environ Mol Mutagen. 2015;56(5):446456.

18. Högstedt B, Karlsson A. The size of micronuclei in human lymphocytes varies according to inducing agent used. Mutat Res. 1985;156(3):229232.

19. Akkuş İ. Free radicals and physiological effects. Konya: Mimoza Press; 2015:32-42.

20. Speit G, Dennog C, Radermacher P, et al. Genotoxicity of hyperbaric oxygen. Mutat Res. 2012;512(2-3):111-119. 